ISSN = 1980-993X - doi:10.4136/1980-993X
Www.ambi-agua.net
E-mail: ambi-agua@agro.unitau.br
Tel.: (12) 3625-4212

\title{
Áreas hidrologicamente sensíveis: teoria e aplicação para a bacia do rio Barigui, $P R$, Brasil \\ (doi:10.4136/ambi-agua.137)
}

\section{Cesar Augusto Crovador Siefert; Irani dos Santos}

Universidade Federal do Paraná - UFPR

E-mail: cesarsiefert@ufpr.br; irani@ufpr.br

\section{RESUMO}

O conceito de área hidrologicamente sensível (AHS) é discutido e aplicado à bacia do rio Barigui $\left(58,5 \mathrm{~km}^{2}\right)$, localizada no município de Almirante Tamandaré, Paraná, Brasil. Compreendem-se como AHS aquelas áreas da bacia hidrográfica que possuem uma maior probabilidade de atingirem o estado de saturação do solo. Foram definidas como AHS as áreas que permanecem saturadas mais de $30 \%$ do tempo. O modelo Topmodel foi utilizado para simular a dinâmica das zonas saturadas na bacia. O modelo pressupõe que a dinâmica da zona saturada pode ser obtida por similaridade hidrológica, dada pela distribuição espacial do índice topográfico, embasando a construção da probabilidade de saturação do solo ao longo da bacia. O Topmodel apresentou uma boa resposta em relação à vazão observada, confirmando bom desempenho em bacias de mesoescala, com clima úmido e relevo suave. As AHS's da bacia do rio Barigui totalizam $17,1 \mathrm{~km}^{2}$ ou $27 \%$ da área total. Considerando-se as AHS's como de interesse à preservação ambiental, obteve-se um acréscimo de 13,2 km² nas áreas de preservação permanente da bacia hidrográfica, obtidas de acordo com o Código Florestal (Lei n 4.771 / 69), delimitando assim um total de $44 \%$ da área da bacia como área de importância ambiental.

Palavras-chave: áreas hidrologicamente sensíveis; área variável de afluência; Topmodel.

\section{Hydrologically sensitive areas: theory and application to Barigui river watershed, PR, Brazil}

\section{ABSTRACT}

The concept of hydrologically sensitive area (HSA) is discussed and applied to Barigui river watershed $\left(58.5 \mathrm{~km}^{2}\right)$, located in Almirante Tamandaré city, Parana State, Brazil. The HSA is considered as the area of the watershed with a higher probability of reaching a soil saturation condition, and is, therefore, defined as the area that remains saturated for more than $30 \%$ of the time. The Topmodel was used to simulate the saturated zone dynamics in the watershed. The model assumes that the saturated zone dynamics can be obtained by hydrological similarity given by the spatial distribution of topographic index, based on the construction of the soil saturation probability in the watershed. The Topmodel showed good results in observed flow data, confirming a high performance in the meso-scale watersheds, with humid climate and smooth relief. The HSA's in the watershed totaling $17.1 \mathrm{~km}^{2}$ or $27 \%$ of the total area. Considering the HSAs as those of interest for environmental preservation, an increase of $13.2 \mathrm{~km}^{2}$ of the permanent preservation areas in the watershed was found according to the Forest Code (Law $n^{\circ} 4.771 / 69$ ). It makes a total of $44 \%$ of the whole watershed environmentally important.

Keywords: hydrologically sensitive areas; variable source area; Topmodel. 


\section{INTRODUÇÃO}

Atualmente a preocupação com a conservação e manutenção de padrões de qualidade mínimos nos recursos hídricos ultrapassa as esferas de cada ciência, fazendo com que o tema seja tratado a partir de uma ótica multidisciplinar. A disponibilidade de água em quantidade e qualidade adequadas pode ser citada como fator limitante ao desenvolvimento da sociedade contemporânea em sua totalidade. A partir da necessidade de se preservar os recursos hídricos, desenvolvem-se meios para quantificar e delimitar possíveis áreas de risco que, identificadas em um processo de planejamento ambiental, devem receber um manejo diferenciado.

As diversas formas de interação da bacia hidrográfica e o ciclo hidrológico possibilitaram o surgimento de múltiplos conceitos explicando os processos e subprocessos decorrentes dessas ações recíprocas. Sendo assim, surge a noção de área variável de afluência (AVA) (Hewlett e Hibbert, 1967), processo observado frequentemente em áreas onde o escoamento superficial por saturação é dominante, tratando o escoamento de retorno e a precipitação incidente nas áreas saturadas como elementos chaves na geração de escoamento.

Considerando que as AVA's possuem uma clara importância no planejamento ambiental, não somente na conservação dos recursos hídricos, mas também por comportarem uma microfauna e uma flora adaptada às variações das condições de saturação dessas áreas, surge a possibilidade de uma intervenção pontual, por meio de práticas de manejo restritivas a usos do solo que comprometam a qualidade dos recursos hídricos. Nesse contexto, surge o conceito de área hidrologicamente sensível (AHS) (Walter et al., 2000), definido como sendo as áreas de uma bacia hidrográfica mais propensas a atingirem o estado de saturação do solo, possibilitando a delimitação das zonas críticas de manejo.

Com o objetivo de simular a dinâmica espacial e temporal das áreas saturadas de uma bacia hidrográfica, o modelo Topmodel (Beven e Kirkby, 1979) considera que o relevo é o elemento da paisagem que controla a dinâmica da água na superfície, exercendo um controle dominante sobre o escoamento superficial e incorporando o conceito de AVA's na sua formulação. Salienta-se ainda que diversos estudos indicam a predominância do escoamento superficial por saturação na região Sul e Sudeste do Brasil, utilizando a modelagem hidrológica para a simulação do processo chuva-vazão a partir do modelo Topmodel (Santos, 2001; Ferreira, 2004; Santos e Kobiyama, 2008; Santos, 2009).

Nesse sentido, destaca-se a utilização de modelos hidrológicos de simulação do processo precipitação vazão, mais precisamente do Topmodel, como importante ferramenta na compreensão da dinâmica das AVA's em bacias hidrográficas e, posteriormente, na definição das AHS's e zonas críticas de manejo.

Dessa forma, propõe-se a utilização do Topmodel como forma de simular os processos de escoamento superficial por saturação na bacia hidrográfica do rio Barigui, possibilitando a delimitação das AHS's.

A bacia hidrográfica do rio Barigui, área de estudo deste trabalho, está localizada integralmente no município de Almirante Tamandaré, Paraná, Brasil. Considera-se que a bacia apresenta uma situação singular dentro da perspectivas de compreensão dos impactos ambientais causados por significativos processos de uso e ocupação do solo devido a suas características rurais e de proximidade à zona de expansão urbana, tornando-se imprescindíveis os estudos que visem identificar as possíveis áreas críticas.

Dentro do exposto, o presente artigo tem como objetivo principal discutir e aplicar o conceito de AHS's para a bacia hidrográfica do rio Barigui e, citam-se ainda como objetivos específicos: determinar a dinâmica das áreas saturadas da bacia, pela simulação do processo chuva-vazão no Topmodel e realizar o mapeamento da bacia, considerando a probabilidade de saturação solo. 


\section{1. Área Variável de Afluência (AVA) e sensibilidade hidrológica}

O conceito de AVA pressupõe que em uma bacia hidrográfica hipotética com uma densa cobertura vegetal, os processos geradores de escoamento superficial não seriam produzidos em toda a extensão da bacia. Salienta-se que o entendimento desse conceito enquanto mecanismo de geração de escoamento por saturação é resultante de distintas pesquisas em bacias experimentais, que ocorreram de forma independente e simultânea na França (Cappus, 1960), Japão (Tsukamoto, 1963) e Estados Unidos (Hewlett e Hibbert, 1967). Diversos trabalhos posteriores consolidaram o conhecimento de que o escoamento superficial seria produzido em uma pequena parte da bacia hidrográfica, cujas dimensões são variáveis no espaço-tempo (Hewlett e Hibbert, 1967; Kirkby, 1969; Dunne, 1970; Dunne e Black, 1970; Dunne et al., 1975). De acordo com essa teoria, essas áreas mais propensas à geração de escoamento superficial por saturação, tendem a expandir-se e contrair-se de forma sazonal, configurando uma parcela de área da bacia variável entre 1\% e 50\% da área total (Dickinson e Whiteley, 1970), dependendo da quantidade e da intensidade de precipitação. Como forma de compreensão desse processo dinâmico, a Figura 1 mostra a variação das áreas saturadas em relação aos diferentes tempos do hidrograma.

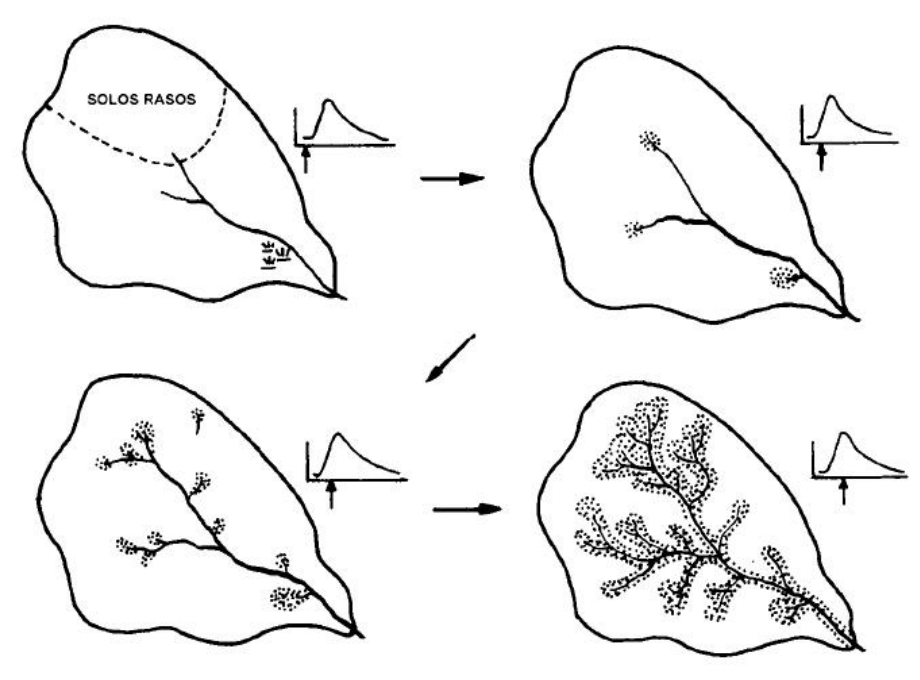

Figura 1. Dinâmica das AVA's no processo de geração de escoamento.

Fonte: Chorley, 1978.

Em bacias de clima úmido e com uma densa cobertura vegetal, o processo de escoamento superficial hortoniano fica restrito a pequenas áreas isoladas na extensão da bacia, onde situações específicas, envolvendo a topografia e condições de baixa permeabilidade do solo permitirão o seu aparecimento. Em um evento de precipitação contínua, na maior parte da área da bacia hidrográfica a capacidade de infiltração será superior a intensidade da chuva, não gerando o processo de escoamento hortoniano. Essa parcela de água infiltrada formará os fluxos subsuperficial e subterrâneo, alimentando os canais e cursos da água. A partir do momento em que a taxa de fluxo subsuperficial exceder a capacidade do solo em transportá-lo ocorre o processo de elevação do nível de água no solo, emergindo a superfície como escoamento de retorno, de importância direta nos processos de escoamento direto, provocando a expansão das AVA's. Sendo assim, o processo de expansão das AVA's está intrinsecamente ligado à extensão das áreas saturadas próximas aos canais perenes em direção aos canais intermitentes, resultantes dos processos desencadeados a partir do evento de precipitação (Hewlett e Hibbert, 1967).

Dessa forma, considera-se que o conceito de AVA está ligado às características físicas da paisagem, enquanto o conceito de AHS (Walter et al., 2000) trata de uma forma de avaliação 
e prevenção de impactos na qualidade da água a partir dos riscos de contaminação dos corpos de água, considerando a probabilidade de geração de escoamento superficial em uma determinada área de uma bacia hidrográfica (Agnew et al., 2006). A importância da compreensão das AHS's é evidente ao tratarmos da gestão dos recursos hídricos, pois essas áreas surgem como um elemento de ligação entre as vertentes e os corpos de água, propiciando o rápido transporte de poluentes através dos processos de escoamento (Walter et al., 2000). Destaca-se ainda a possibilidade de uma intervenção pontual nessas áreas de risco, servindo também como uma maneira de prever possíveis desastres ambientais a partir do mapeamento das zonas críticas de manejo. Assim a noção de AHS's está intimamente relacionada ao funcionamento das AVA's de uma bacia hidrográfica e ao uso do solo nesses locais, podendo servir de subsídio ao planejamento ambiental.

A partir da ideia de que formas de poluição difusa são causas de alteração e degradação na qualidade dos corpos de água e de que as práticas conhecidas de manejo e conservação de solo e recursos hídricos, definidas na Lei Federal 4.771/69 que institui o Código Florestal Brasileiro e suas posteriores modificações, não levam explicitamente em consideração os processos hidrológicos, a proposta do conceito de AHS's é uma forma de buscar cientificamente a compreensão dos processos geradores de escoamento superficial como medida preventiva a ser adotada na conservação de solos e manutenção da qualidade dos corpos de água. Walter et al. (2000) afirmam que uma quantificação hidrológica fornece o método mais apropriado para avaliar o risco de contaminação dos corpos de água. Assim, o conceito de AHS's aliado à noção de AVA's tem implicações diretas na conservação dos recursos hídricos, contribuindo para uma gestão sustentável e diminuindo riscos de contaminação dos mananciais por poluição difusa provenientes, principalmente, de práticas agrícolas e/ou de pecuária inadequadas.

Em bacias hidrográficas onde o processo de AVA é o dominante na geração de escoamento superficial, as áreas com uma maior probabilidade de gerar escoamento podem ser consideradas como sendo AHS's. Nesse caso, observa-se o limite de sensibilidade hidrológica $\left(\mathrm{L}_{A H S}\right)$ ao longo da bacia hidrográfica, analisada a partir da probabilidade de saturação $\left(\mathrm{P}_{\text {sat }}\right)$, para delimitação espacial das AHS's. Devido à alta capacidade de geração de escoamento superficial, aliada a uma potencial característica de transporte de poluentes, a poluição incidente nessas áreas tende a escoar diretamente os corpos de água, estabelecendo zonas críticas de manejo (Figura 2). Considera-se uma AHS da bacia hidrográfica como sendo uma zona crítica de manejo quando as práticas de uso do solo diretamente sobre elas possam vir a degradar a qualidade dos corpos hídricos.

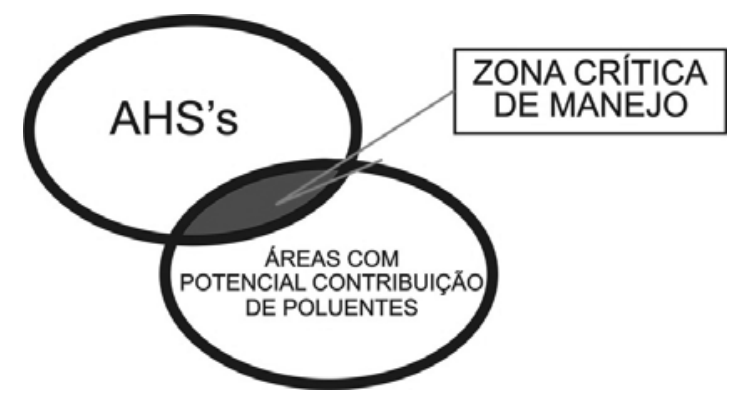

Figura 2. Representação das zonas críticas de manejo.

Fonte: adaptado de Walter et al. (2001).

Em trabalhos recentes (Tabela 1), os conceitos de AHS's e zonas críticas de manejo foram adotados com o objetivo de orientar práticas agrícolas e de pecuária no sentido de diminuir substancialmente o nível de contaminação dos corpos hídricos por poluição difusa 
SIEFERT, C. A. C.; DOS SANTOS, I. Áreas hidrologicamente sensíveis: teoria e aplicação para a bacia do rio Barigui, PR, Brasil. Ambi-Agua, Taubaté, v. 5, n. 2, p. 61-76, 2010. (doi:10.4136/ambi-agua.137)

causada pelo manejo inadequado de fertilizantes (Walter et al., 2000; Walter et al., 2001; Gburek et al., 2002; Agnew et al., 2006; Gorsevski et al., 2008).

Tabela 1. Exemplos da utilização dos conceitos de AHS’s e zonas críticas de manejo.

\begin{tabular}{|c|c|c|c|c|}
\hline Localização & $\begin{array}{l}\text { Área } \\
\left(\mathbf{k m}^{2}\right)\end{array}$ & Uso do Solo & Descrição & Referências \\
\hline $\begin{array}{l}2 \text { Sub-bacias - Catskill } \\
\text { Mountains, New York } \\
\text { - EUA. }\end{array}$ & $\begin{array}{c}40,1 \text { e } \\
93,8\end{array}$ & $\begin{array}{l}\text { Destinado à criação } \\
\text { de gado leiteiro. }\end{array}$ & $\begin{array}{c}\text { Determinar limite de sensibilidade } \\
\text { hidrológica, } \mathrm{P}_{\text {sat }}>30 \% \text {. }\end{array}$ & $\begin{array}{l}\text { Walter et al. } \\
\text { (2000) }\end{array}$ \\
\hline $\begin{array}{l}\text { Arredores de Catskill } \\
\text { Mountains, New York } \\
\text { - EUA }\end{array}$ & 1,43 & $\begin{array}{l}\text { Destinado à criação } \\
\text { de gado leiteiro. }\end{array}$ & $\begin{array}{c}\text { Teste de cenário, considerando a época } \\
\text { do ano para determinar a relação } \mathrm{P}_{\text {sat }} \text { e } \\
\text { AHS's }\end{array}$ & $\begin{array}{l}\text { Walter et al. } \\
\text { (2001) }\end{array}$ \\
\hline $\begin{array}{l}\text { Bacia piloto FD-36, } \\
\text { Pennsylvania - EUA }\end{array}$ & 0,395 & $\begin{array}{l}\text { Misto (pastagens, } \\
\text { milho, soja, feijão e } \\
\text { florestas). }\end{array}$ & $\begin{array}{l}\text { Metodologia que inclui o “índice de } \\
\text { fósforo” ao conceito de AVA’s, com o } \\
\text { objetivo de determinar zonas críticas de } \\
\text { manejo. }\end{array}$ & $\begin{array}{l}\text { Gburek et al. } \\
\text { (2002) }\end{array}$ \\
\hline $\begin{array}{l}3 \text { bacias-piloto, New } \\
\text { York - EUA }\end{array}$ & $\begin{array}{c}1,6 \mathrm{a} \\
37\end{array}$ & $\begin{array}{l}\text { Pastagens e } \\
\text { florestas. }\end{array}$ & $\begin{array}{l}\text { Teste comparativo da variação das } \\
\text { AHS’s a partir do valor de } \mathrm{P}_{\text {sat }}>5 \% \text {, } \\
\text { obtidas por dois parâmetros distintos. }\end{array}$ & $\begin{array}{l}\text { Agnew et al. } \\
\text { (2006) }\end{array}$ \\
\hline $\begin{array}{l}\text { Bacia hidrográfica de } \\
\text { Pete King, Idaho - } \\
\text { EUA }\end{array}$ & 72 & Florestas & $\begin{array}{l}\text { Proposta de buffers de conservação } \\
\text { dinâmicos, a partir do conceito de } \\
\text { AHS’s e zonas prioritárias de manejo }\end{array}$ & $\begin{array}{l}\text { Gorsevski et } \\
\text { al. (2008) }\end{array}$ \\
\hline
\end{tabular}

Assim, torna-se importante a incorporação de conhecimento de processos hidrológicos nas práticas de manejo do solo ou por meio de restrições de uso em determinadas épocas do ano e em determinadas áreas da bacia, uma vez que a dinâmica das AVA's faz com que as AHS's também sejam variáveis no espaço-tempo. Uma das formas eficazes de determinação das AHS's é a utilização de modelos hidrológicos que possam determinar as áreas com maior probabilidade na geração de escoamento superficial por saturação em uma bacia hidrográfica, visando a ações preventivas diretamente nas zonas críticas de manejo.

\section{MATERIAL E MÉTODOS}

A área de estudo compreende a bacia de drenagem do rio Barigui, a montante da estação hidroambiental Almirante Tamandaré, com área de drenagem de 58,5 km², situada entre as coordenadas $25^{\circ} 23^{\prime}$ e $25^{\circ} 32^{\prime}$ de latitude sul e $49^{\circ} 29^{\prime}$ e $49^{\circ} 33^{\prime}$ de longitude oeste. A bacia hidrográfica está totalmente inserida no município de Almirante Tamandaré, situado na Região Metropolitana de Curitiba, no estado do Paraná.

As AHS's da bacia do rio Barigui foram determinadas a partir da simulação do processo chuva-vazão com o modelo hidrológico Topmodel (Beven e Kirkby, 1979). Um maior detalhamento sobre o funcionamento e equacionamento do modelo pode ser encontrado em Beven et al. (1995); Mine e Clark (1996); Varella e Campana (2000); Santos (2001); Silva e Kobiyama (2007) e Santos (2009).

O Topmodel é um modelo de transformação de chuva em vazão do tipo conceitual determinístico, semidistribuído e de base física, que considera o conceito de AVA na formação de escoamento superficial (Varella e Campana, 2000).

Os dados hidrológicos de entrada necessários para a simulação do Topmodel são: precipitação, evapotranspiração potencial e vazão observada. No presente trabalho, optou-se pela utilização de dados horários, conforme recomendado em Agnew et al. (2006). O modelo ainda utiliza dados relativos às condições geomorfológicas da bacia hidrográfica distribuídos espacialmente, sendo: a distribuição do índice topográfico (IT) e a relação entre a área drenada e o comprimento do canal principal. A versão do Topmodel utilizada (97.01) possui 
cinco parâmetros de entrada que exigem a calibração com valores médios para toda a área da bacia hidrográfica simulada. A Tabela 2 apresenta a descrição dos parâmetros do Topmodel.

Tabela 2. Descrição dos parâmetros de entrada do Topmodel.

\begin{tabular}{c|l|c}
\hline Parâmetro & \multicolumn{1}{|c}{ Descrição } & Unidade \\
\hline$m$ & Função da transmissividade exponencial & $\mathrm{m}$ \\
In(To) & Logaritmo natural de transmissividade do solo saturado & $\mathrm{m}^{2} / \mathrm{h}$ \\
SRMax & Armazenamento de água na zona das raízes & $\mathrm{m}$ \\
SRInit & Déficit de armazenamento inicial na zona das raízes & $\mathrm{m}$ \\
ChVel & Velocidade de propagação do escoamento no canal & $\mathrm{m} / \mathrm{h}$ \\
\hline
\end{tabular}

Para o Topmodel a probabilidade de saturação de um pixel qualquer da bacia é determinada pelo índice topográfico. O índice topográfico representa a propensão de um ponto qualquer da bacia atingir o estado de saturação (Schuler et al., 2000), e sua distribuição espacial indicará as áreas com maior potencial para gerar escoamento superficial por saturação. Outra questão interessante sobre o índice topográfico é o princípio da similaridade hidrológica, ou seja, todas as áreas que possuem o mesmo valor de índice topográfico apresentam um comportamento hidrológico similar, quanto maior o valor do índice topográfico maior a probabilidade de atingir o estado de saturação. O IT é dado pela relação entre a área específica de contribuição até o pixel por unidade de largura do contorno - $a(\mathrm{~m})$ pela tangente da declividade do terreno no pixel $-\operatorname{tg} \beta(\mathrm{m} / \mathrm{m})$.

$$
I T=\ln (a / \operatorname{tg} \beta)
$$

O mapa da distribuição espacial do IT foi obtido a partir do modelo digital do terreno hidrologicamente consistido (Lopes e Santos, 2009), com resolução espacial de $5 \mathrm{~m}$, construído a partir de cartas topográficas obtidas do levantamento altimétrico realizado para a área de estudo na escala 1:10.000 (SUDERHSA-PR, 2000).

Os valores de índice topográfico estão coerentes com as características físicas da bacia, uma vez que áreas com elevadas declividades e/ou pequena área de contribuição apresentam um baixo valor de índice topográfico. Nota-se que, aproximadamente, 85\% dos pixels da bacia hidrográfica apresentam um valor de IT $<8,0$.

A Figura 3 mostra a distribuição do índice topográfico em relação à porcentagem da área da bacia do rio Barigui e a Figura 4 mostra a distribuição espacial do IT, e as áreas com maior valor do índice são mais propensas a atingirem o estado de saturação.

As AHS's foram definidas a partir da estimativa da probabilidade de geração de escoamento superficial por saturação, ou seja, a probabilidade de essa área atingir o estado de saturação durante um evento de precipitação. Para expressar esse conceito, AHS's são quaisquer áreas $(A)$ em que a probabilidade $(P)$ de serem consideradas sensíveis é maior que o Limite de Sensibilidade Hidrológica $\left(L_{A H S}\right)$, conforme definido por Walter et al., (2000), expresso na relação:

$$
A^{1}=\text { AHS's se } P\left(A^{1}=\text { sensível }\right)>\left(L_{A H S}\right)
$$


SIEFERT, C. A. C.; DOS SANTOS, I. Áreas hidrologicamente sensíveis: teoria e aplicação para a bacia do rio Barigui, PR, Brasil. Ambi-Agua, Taubaté, v. 5, n. 2, p. 61-76, 2010. (doi:10.4136/ambi-agua.137)

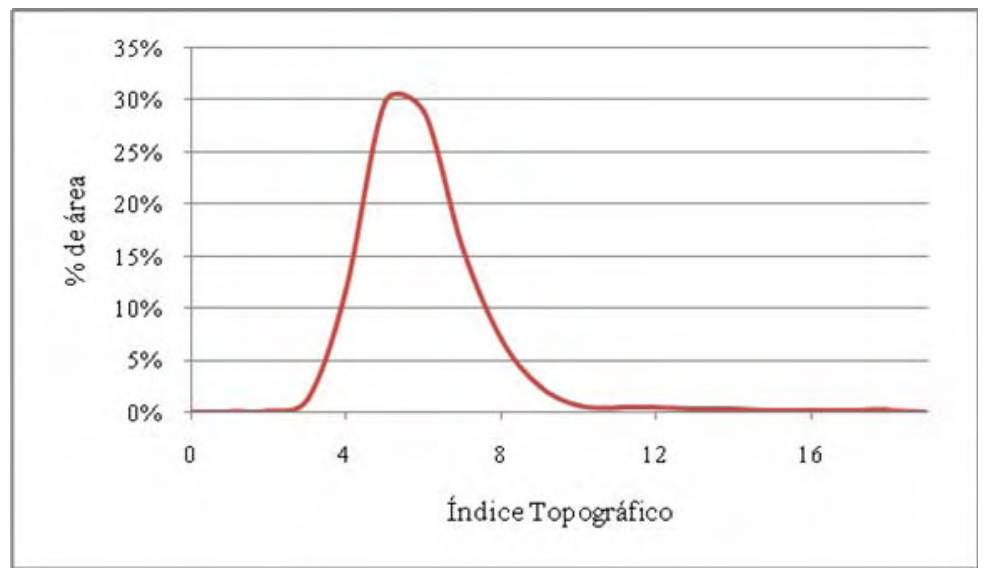

Figura 3. Distribuição do IT para a bacia do rio Barigui em relação a \% da área total.

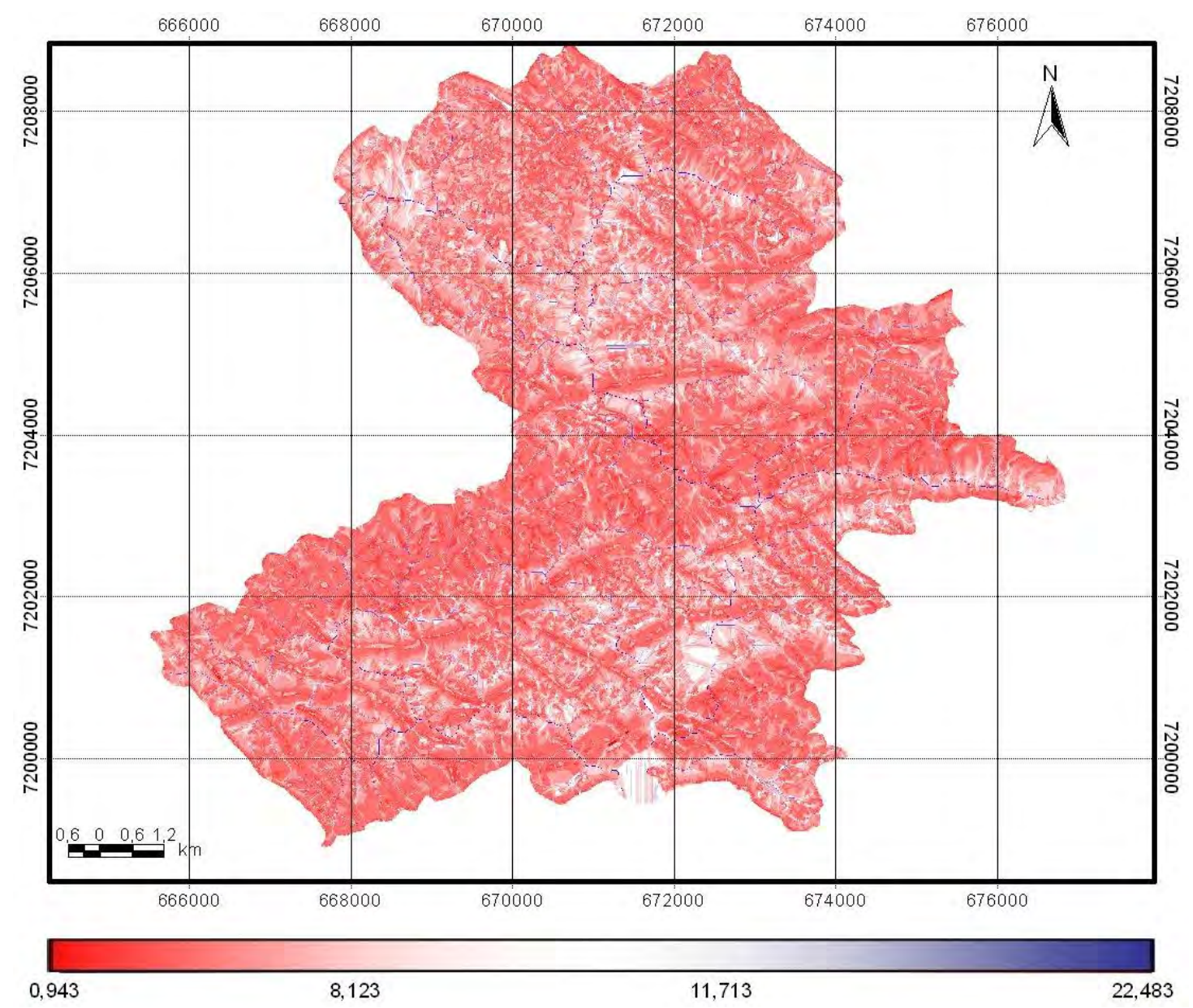

Figura 4. Distribuição espacial do IT para a bacia do rio Barigui.

De uma maneira simplificada, a delimitação das AHS's necessita do cruzamento das seguintes informações: [1] distribuição espacial do índice topográfico da área de estudo; e [2] probabilidade de saturação $\left(\mathrm{P}_{s a t}\right)$ de cada pixel da área considerada.

$\mathrm{O}$ índice $\mathrm{P}_{\text {sat }}$ foi estimado a partir da distribuição espacial da dinâmica das áreas saturadas no tempo considerado para a simulação, geradas pelo Topmodel. Com a compilação desses dados, pode-se construir um gráfico correlacionando a porcentagem da área da bacia que atinge o estado de saturação pelo tempo, ou seja, uma curva de permanência das áreas saturadas da bacia hidrográfica. 
Essa curva de permanência foi utilizada juntamente com o IT em ambiente SIG, com o objetivo de mapear a probabilidade de saturação da bacia considerada. Utilizando-se o software ArcView 3.2, realizou-se o cruzamento do mapa da distribuição do IT com as informações da porcentagem de área saturada da bacia no tempo, possibilitando a construção de um mapa que representasse a probabilidade de cada pixel da bacia hidrográfica atingir o estado de saturação durante o período de tempo simulado.

Obteve-se dessa forma, um mapa indicativo da $\mathrm{P}_{\text {sat }}$ por pixel da bacia, que subsidiou a determinação das AHS's. Ou seja, para o mapeamento das AHS's, deve-se efetuar o cruzamento da $\mathrm{P}_{\text {sat }}$ de cada pixel com a distribuição espacial do IT, visando mapear as AHS's na bacia hidrográfica, conforme metodologia esquematizada na Figura 5.

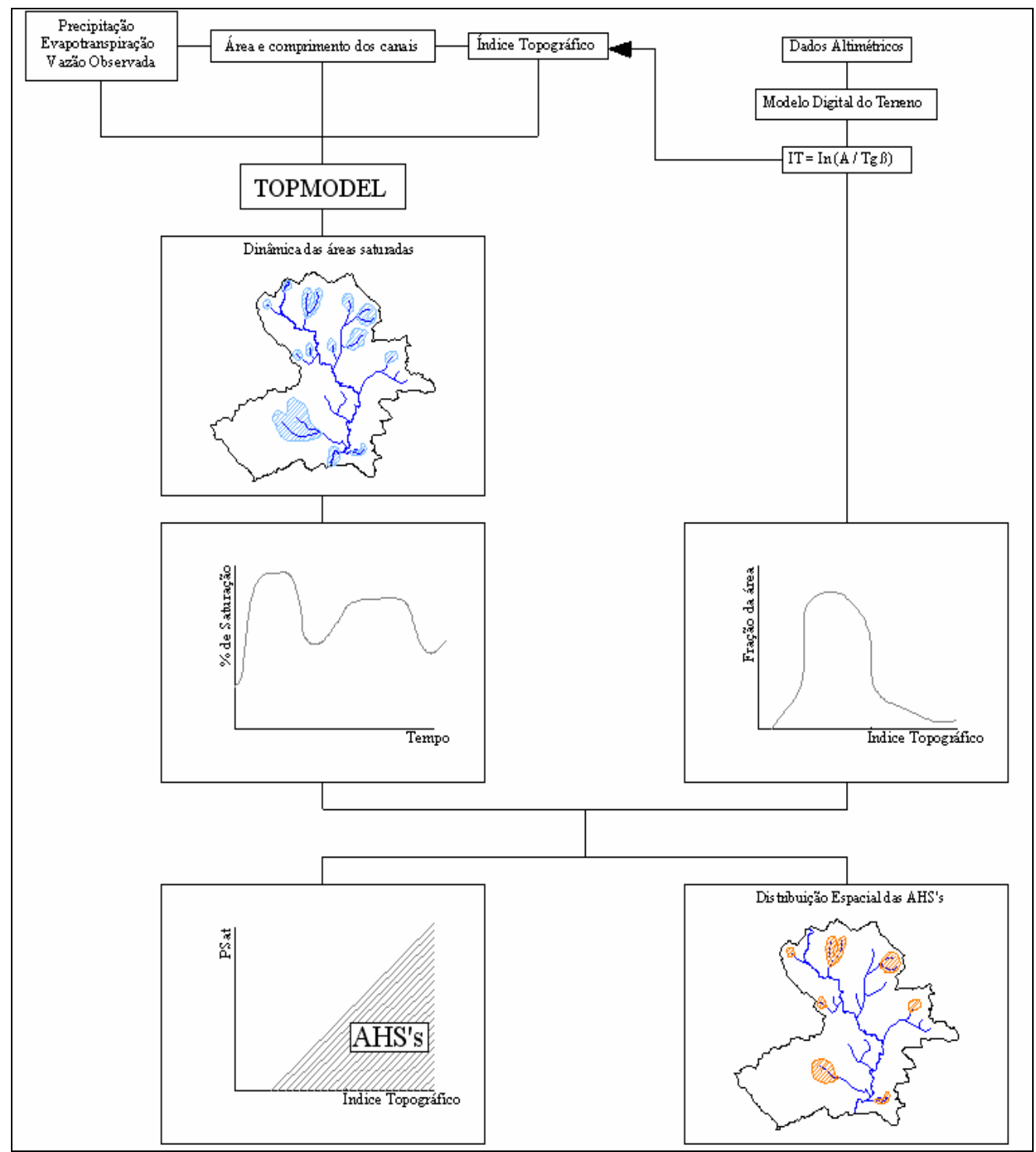

Figura 5. Metodologia para delimitação das AHS’s.

\section{RESULTADOS E DISCUSSÃO}

A simulação hidrológica foi realizada para o período compreendido entre 17/09/2002 a 16/12/2002, totalizando 2.184 horas. A calibração do Topmodel foi realizada diretamente no modelo, de maneira semiautomática visando maximizar o coeficiente E proposto por Nash e Sutcliffe (1970). Valores mais próximos de 1 indicam um melhor ajuste dos valores observados e simulados. Mine e Clarke (1996) citam que para uma simulação aceitável, o 
valor de E deve ser igual ou superior a 0,7. O valor de E obtido na simulação, assim como os parâmetros utilizados são apresentados na Tabela 3.

Tabela 3. Valores dos parâmetros calibrados no Topmodel para a bacia do rio Barigui.

\begin{tabular}{c|cccccc}
\hline Período & $\boldsymbol{M}$ & $\mathbf{I n}($ To $)$ & SRmax & SRInit & ChVel & E \\
\hline $17 / 09 / 2002-16 / 12 / 2002$ & 0,007 & $-2,20$ & 0,005 & 0,0018 & 1230 & 0,781 \\
\hline
\end{tabular}

As vazões simuladas mostraram-se coerentes aos dados de vazões observadas na estação hidroambiental Almirante Tamandaré em relação aos picos do hidrograma simulado. Entretanto, destaca-se que, nas recessões, o modelo apresentou limitação para reproduzir os dados observados (Figura 6). Essa dificuldade em simular adequadamente as recessões pode estar relacionada à ocorrência na bacia de processos hidrológicos não contemplados nas hipóteses simplificadoras do modelo (Beven, 2002), como, por exemplo, a existência de escoamento subsuperficial por caminhos preferenciais ou ausência de conectividade entre as áreas saturadas (Santos, 2009).

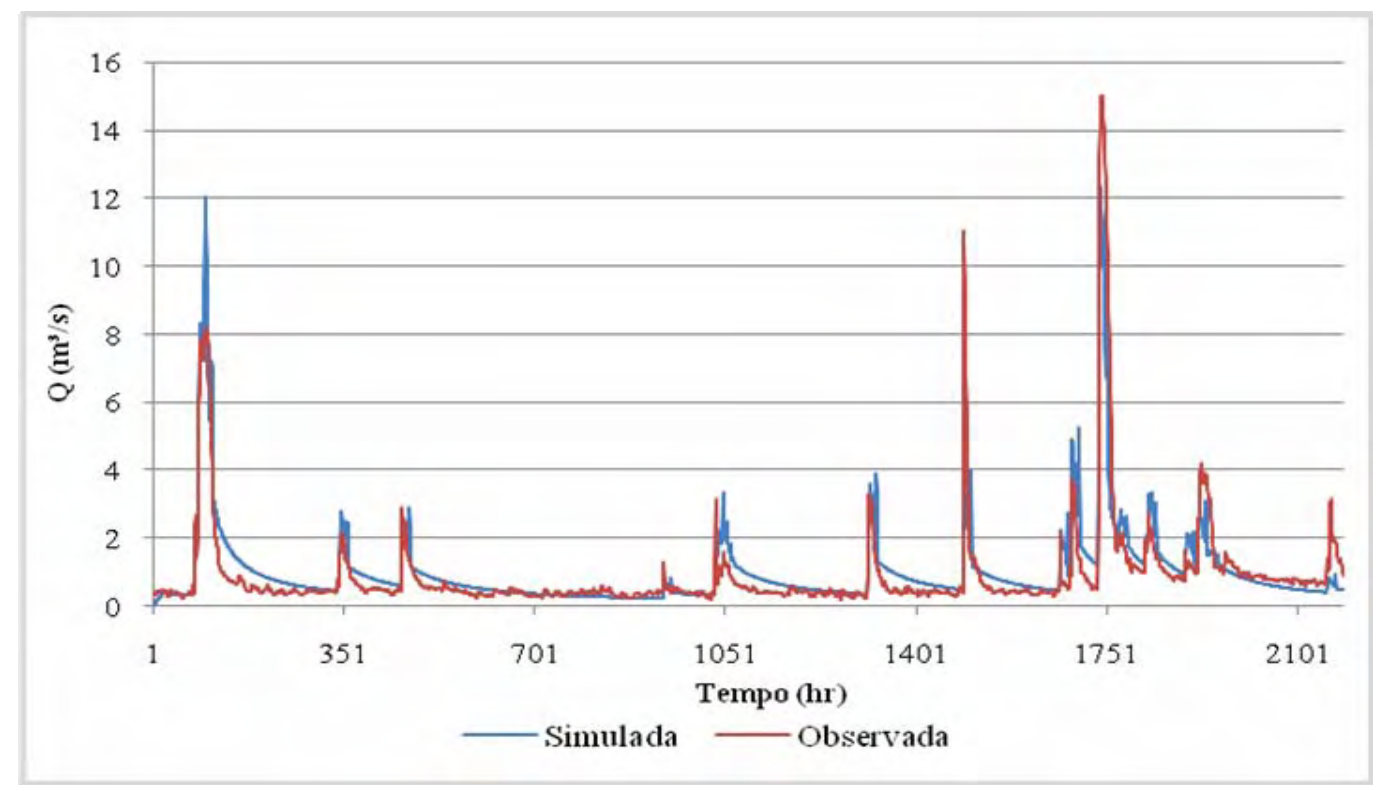

Figura 6. Hidrograma das vazões simuladas e observadas para a bacia do rio Barigui.

Da simulação do Topmodel resultou ainda a dinâmica das áreas saturadas na bacia hidrográfica durante o período considerado. Dessa forma, obtiveram-se 2.184 mapas que reproduzem a variação espaço-temporal das áreas saturadas. Durante o período de 17/09/2002 a 16/12/2002, observou-se uma variação da porcentagem de área saturada na bacia entre 6,1\% a 46,1\%. A dinâmica das áreas saturadas no tempo é mostrada na Figura 7. A distribuição espacial das áreas saturadas mínimas e máximas pode ser observada na Figura 8. 
SIEFERT, C. A. C.; DOS SANTOS, I. Áreas hidrologicamente sensíveis: teoria e aplicação para a bacia do rio Barigui, PR, Brasil. Ambi-Agua, Taubaté, v. 5, n. 2, p. 61-76, 2010. (doi:10.4136/ambi-agua.137)

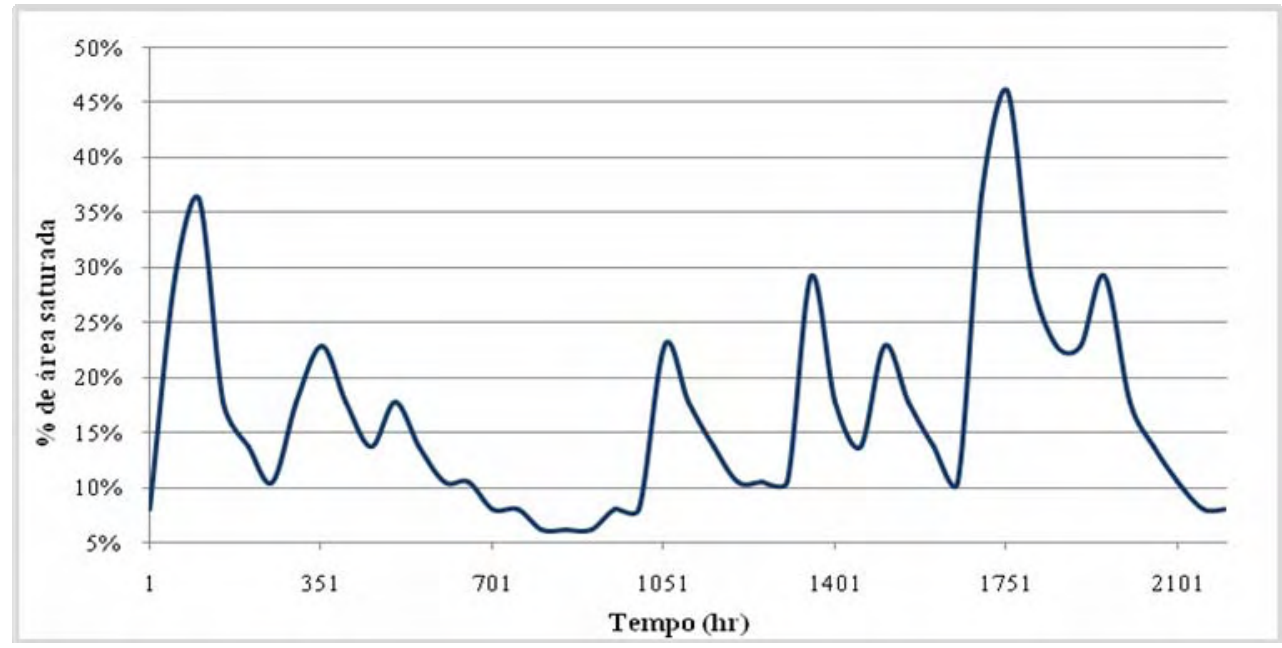

Figura 7. Dinâmica das áreas saturadas para a bacia do rio Barigui.

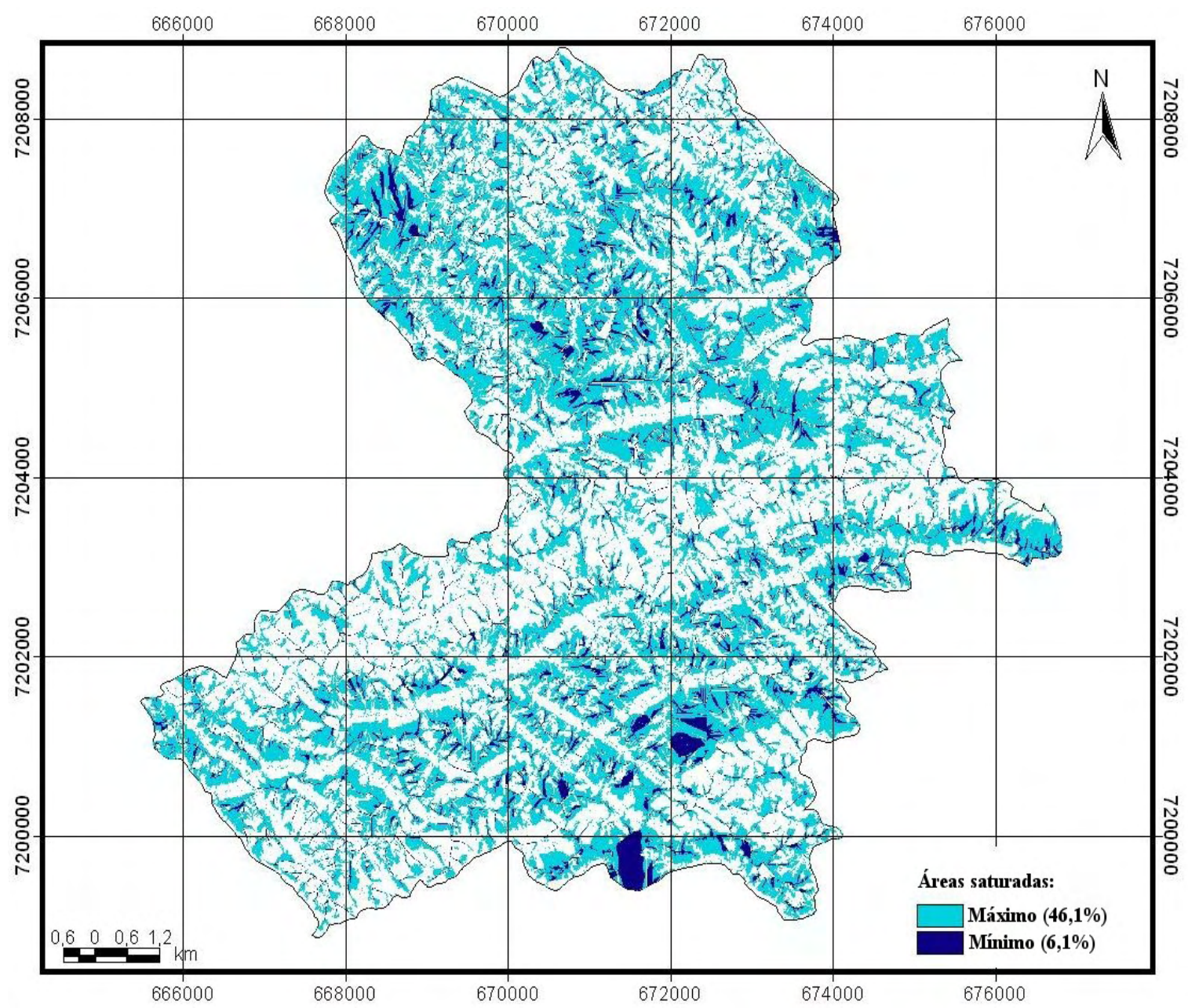

Figura 8. Áreas saturadas máximas e mínimas para a bacia do rio Barigui.

Devido à ausência de sazonalidade hidrológica na bacia hidrográfica do rio Barigui (Fill et al., 2005), a dinâmica da probabilidade de saturação da bacia manteve um comportamento similar entre os meses simulados, resultando em pequena variação no comportamento da $\mathrm{P}_{\text {sat }}$. A Figura 9 mostra a relação entre $\mathrm{P}_{\text {sat }}$ e IT, a área preenchida indica os valores de IT que permaneceram saturados durante o período simulado. 
SIEFERT, C. A. C.; DOS SANTOS, I. Áreas hidrologicamente sensíveis: teoria e aplicação para a bacia do rio Barigui, PR, Brasil. Ambi-Agua, Taubaté, v. 5, n. 2, p. 61-76, 2010. (doi:10.4136/ambi-agua.137)

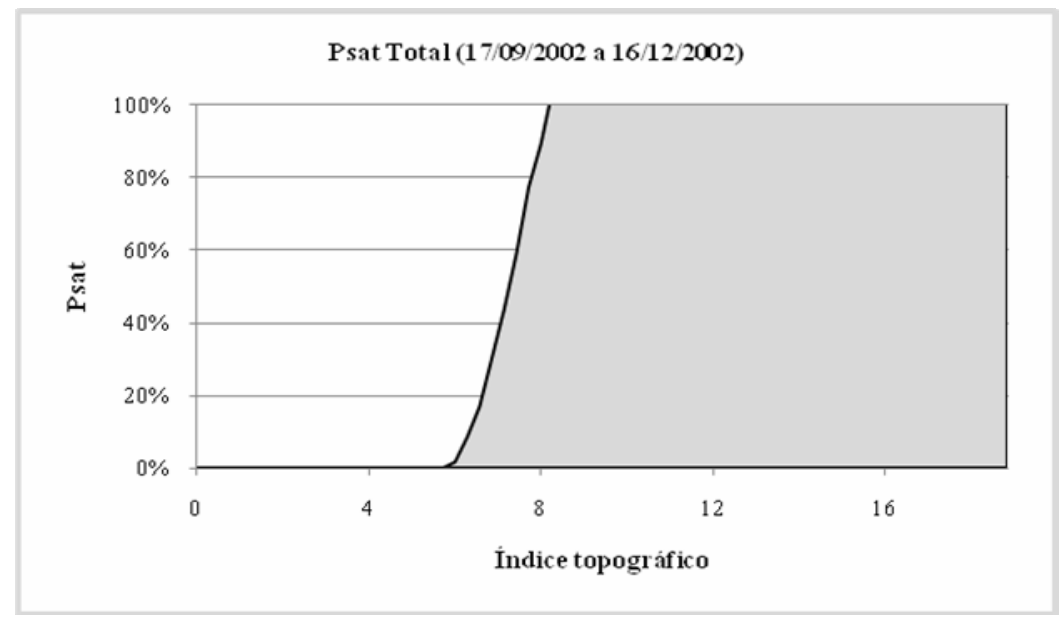

Figura 9. $\mathrm{P}_{\text {sat }}$ em relação à distribuição do IT para a bacia do rio Barigui.

Nota-se que a variação da $\mathrm{P}_{\text {sat }}$ para o período simulado compreende o intervalo de IT entre 6,638 e 8,143; ou seja, durante o período simulado as áreas da bacia que apresentam um valor de IT > 8,143 encontraram-se permanentemente saturadas, o que corresponde a uma área mínima saturada de $6,1 \%$ da bacia. No evento de maior precipitação, as áreas que apresentam um valor de IT > 6,638 atingiram o estado de saturação, contabilizando o valor de 46,1\% de área saturada máxima.

Com base no exposto acima sobre a variação da $\mathrm{P}_{\text {sat }}$ durante as 2.184 horas simuladas, pôde-se realizar o cruzamento da $\mathrm{P}_{\text {sat }}$ total com os valores de índice topográfico, de modo que possibilite obter a distribuição espacial das AHS's. Realizou-se a compilação da $\mathrm{P}_{\text {sat, }}$ resultando em um mapa que indica, em relação ao tempo total, a porcentagem de tempo que cada pixel permanece saturado, conforme mostrado na Figura 10.

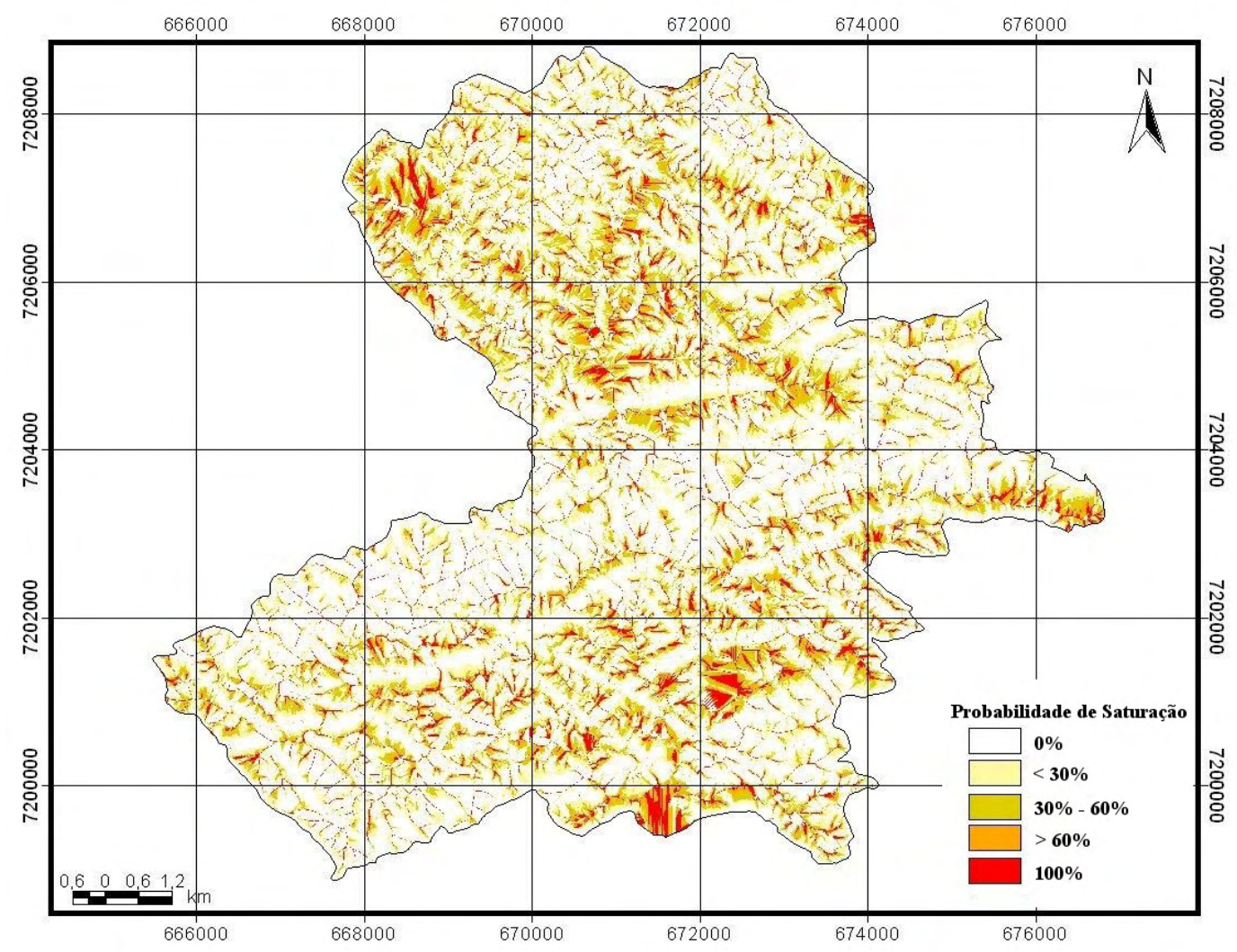

Figura 10. Mapa da $\mathrm{P}_{\text {sat }}$ da bacia do rio Barigui. 
SIEFERT, C. A. C.; DOS SANTOS, I. Áreas hidrologicamente sensíveis: teoria e aplicação para a bacia do rio Barigui, PR, Brasil. Ambi-Agua, Taubaté, v. 5, n. 2, p. 61-76, 2010. (doi:10.4136/ambi-agua.137)

Para a delimitação das AHS's da bacia hidrográfica do rio Barigui adotou-se como $\mathrm{L}_{A H S}$ o valor de $P_{\text {sat }}>30 \%$, de acordo com o recomendado por Walter et al. (2000). Dessa forma considerou-se como AHS's toda a área da bacia que permaneça saturada mais de $30 \%$ do tempo.

A Figura 11 mostra a curva de permanência de $\mathrm{P}_{\text {sat }}$ em relação à porcentagem da área da bacia que atinge o estado de saturação, com indicação do $\mathrm{L}_{A H S}$ adotado. A Figura 12 mostra a distribuição espacial das AHS’s da bacia do rio Barigui.

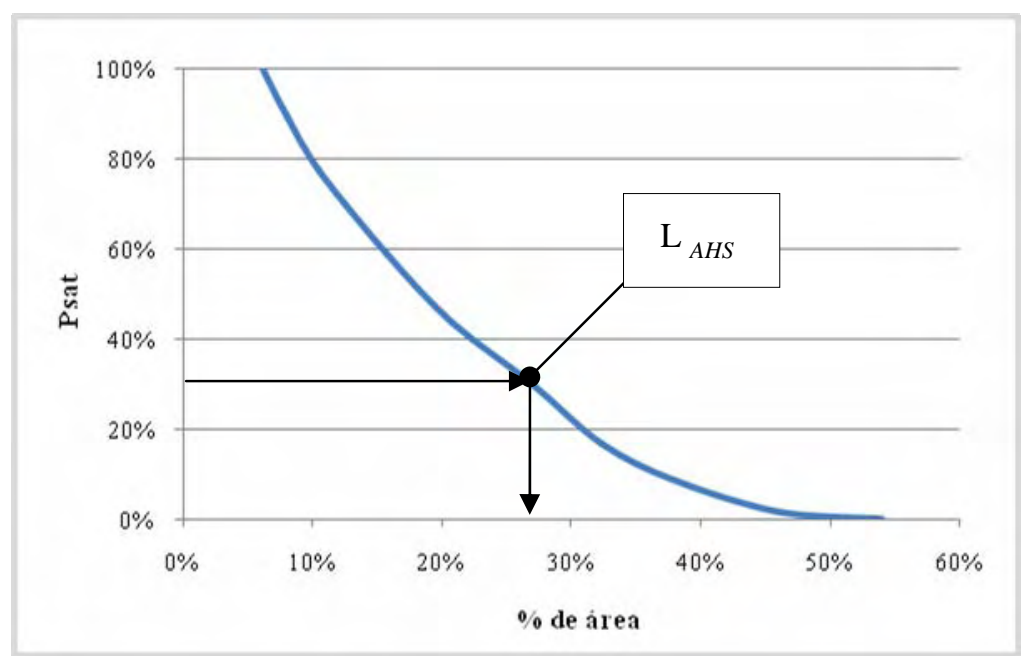

Figura 11. $\mathrm{P}_{\text {sat }}$ em relação à porcentagem de área saturada para a bacia do rio Barigui.

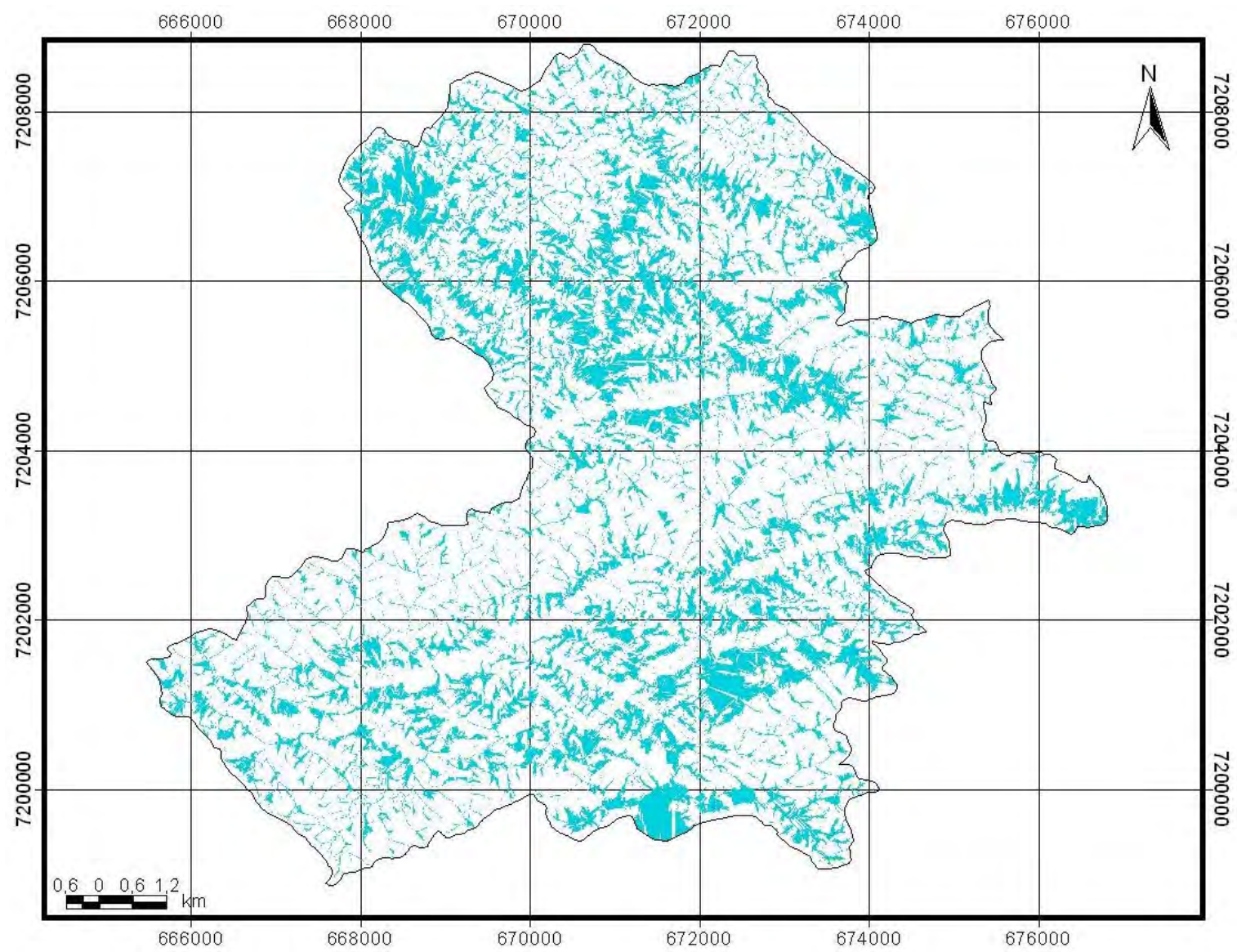

Figura 12. Localização das AHS’s da bacia do rio Barigui. 
Segundo o critério utilizado, as AHS's da bacia hidrográfica do rio Barigui totalizam $17,1 \mathrm{~km}^{2}$, correspondendo a $27,2 \%$ da área total da bacia. O mapeamento das AHS's possibilita realizar uma análise das condições ambientais da área, unindo-se essa informação aos resultados do mapeamento das áreas de preservação da mata ciliar de acordo com o Código Florestal.

As áreas de preservação permanente (APP's) ao longo dos cursos de água da bacia hidrográfica do rio Barigui, obtidas de acordo com a Lei Federal 4.771 / 65, correspondem a 21,4\% da área total da bacia, contemplando $12,5 \mathrm{~km}^{2}$. Com a comparação dos mapas de AHS's e APP's, observada na Figura 13, nota-se que, na maior parte, ambas não coincidem espacialmente. Isso se torna relevante, considerando que as APP’s visam proteger as áreas mais sensíveis da bacia hidrográfica ao longo dos cursos de água. Salienta-se, ainda, que AHS's surgem como elementos de ligação entre os corpos hídricos e as vertentes, destacandose que a não preservação dessas áreas possibilita o surgimento das zonas críticas de manejo, provocando o carreamento de contaminantes potencialmente existentes nessas áreas diretamente para os rios.

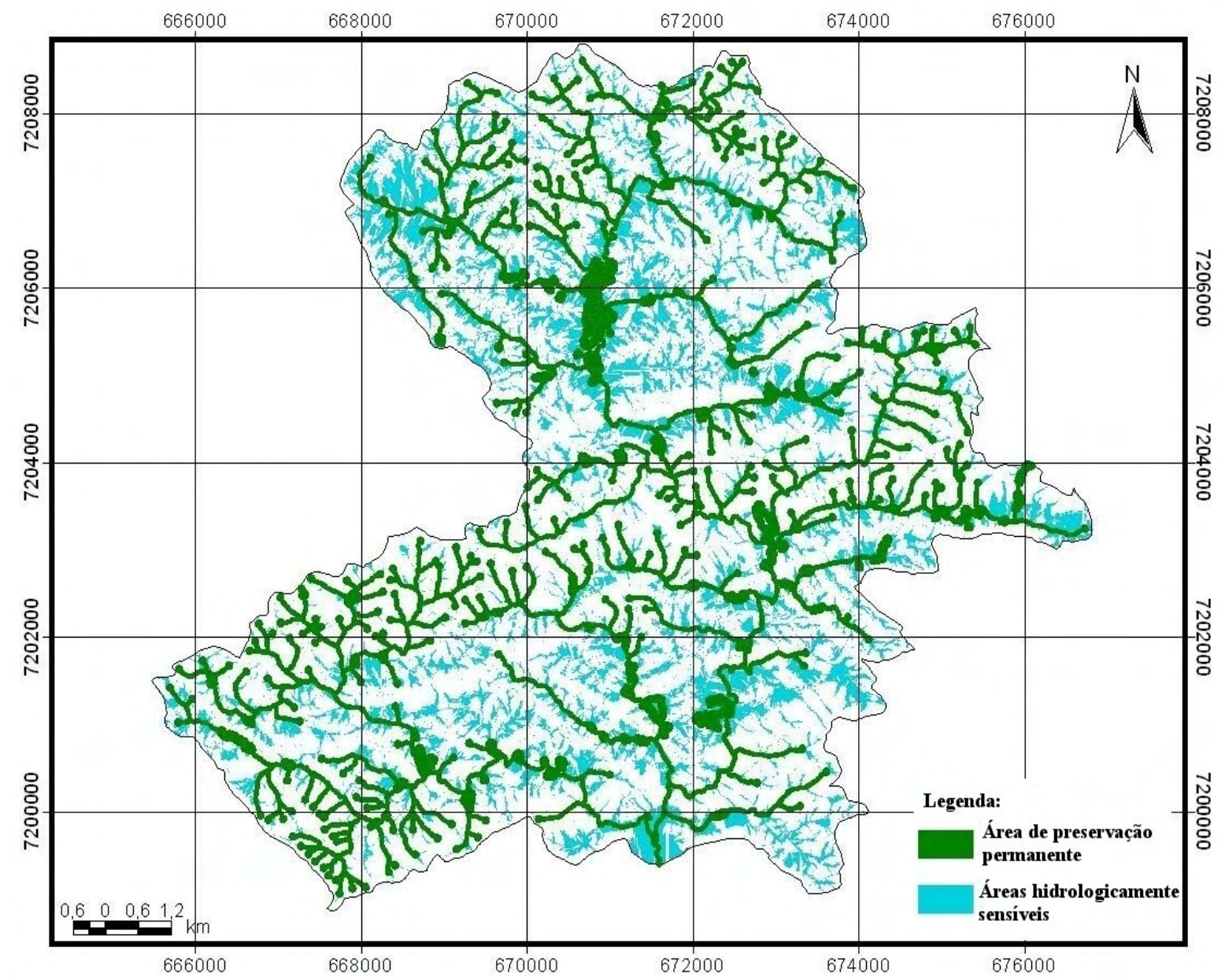

Figura 13. Comparativo entre as AHS's e as APP's da bacia do Rio Barigui.

Considerando a importância da preservação ambiental e a não-coincidência espacial das APP’s e AHS's, da área inicial de 12,5 km² (21,4\%), equivalente às áreas de preservação permanente de mata ciliar, somam-se ainda 13,2 $\mathrm{km}^{2}$ (22,6\%) referentes às áreas em que as AHS's não coincidem com as áreas de preservação permanente. Assim, têm-se 25,7 km² a serem considerados como áreas de importância ambiental na bacia hidrográfica do rio Barigui, totalizando 44\% da sua área total. 
A necessidade da preservação das AHS's deriva da alta probabilidade de saturação, atuando como elemento de conectividade hidrológica entre a paisagem e os corpos de água. Evidentemente que toda prática de manejo inadequada nessas áreas acarretará alterações significativas no equilíbrio dinâmico estabelecido entre os elementos naturais, considerando a existência de condições específicas de biodiversidade nessas áreas, uma vez que essa dinâmica das águas faz com que ocorra uma adaptação necessária a essa condição ambiental. Portanto, destaca-se a importância das AHS's no sistema bacia hidrográfica, devendo ser considerada a necessidade de preservação dessas áreas.

\section{CONCLUSÕES}

Aplicando o conceito de AHS's para a bacia hidrográfica do rio Barigui obteve-se um acréscimo significativo à área de preservação permanente, considerando $44 \%$ da área total da bacia hidrográfica como sendo áreas de sensibilidade ambiental, justificando a necessidade de preservação e restrição quanto ao uso do solo.

O Topmodel respondeu de forma satisfatória às características da bacia hidrográfica do rio Barigui. Isso confirma a potencialidade de utilização do modelo na simulação das AHS's principalmente em bacias de média-escala com clima úmido e relevo suavemente ondulado.

Neste trabalho o conceito de AHS's, pioneiramente aplicado em condições brasileiras, demonstra um grande potencial de utilização no gerenciamento de bacias hidrográficas a partir do entendimento da dinâmica hidrológica local.

A título de recomendação destaca-se que o $\mathrm{L}_{A H S}$ utilizado de $\mathrm{P}_{\text {sat }}>30 \%$, adotado a partir da literatura, seja objeto de investigação futura visando construir um maior embasamento científico a partir de pesquisas em condições brasileiras.

\section{AGRADECIMENTOS}

O primeiro autor, Cesar Augusto Crovador Siefert, agradece a CAPES pela concessão da bolsa de Mestrado.

\section{REFERÊNCIAS}

AGNEW, L. J.; LYON S.; GERARD-MARCHANT P.; COLLINS V. B.; LEMBO A. J.; STEENHUIS T. S.; et al. Identifying hydrologically sensitive areas: bridging the gap between science and application. Journal of Environmental Management, Amsterdam, v. 78, p. 63-76, 2006.

BEVEN, K. J.; KIRKBY, M. J. A physically based, variable contributing area model of basin hydrology. Hydrological Sciences Bulletin, v. 24, p.43-69, 1979.

BEVEN, K. J.; LAMB, R.; QUINN, P.; ROMANOWICIZ, R.; FREER, J. Topmodel. In: SINGH, V. P. (Ed.). Computer models of watershed hydrology. Baton Rouge: Water Resource Publ., 1995. p. 627-668.

BEVEN, K. Towards an alternative blueprint for a physically based digitally simulates hydrologic response modeling system. Hydrological Process, v. 16, p. 189-206, 2002.

BRASIL. Lei $\mathbf{n}^{\circ}$ 4.771, de 15 de setembro de 1965. Institui o novo Código Florestal. Disponível em: <http://www.planalto.gov.br/ccivil_03/Leis/L4771.htm>. Acesso: 11 jun. 2010. 
CAPPUS, P. Bassin experimental d'Alrance: etudes dês lois de l'ecoulement. Application au calcul et a la prevision des debits. La Houille Blanche, v. A, p. 493-514, 1960.

CHORLEY, R. J. The hillslope hydrological cycle. In: KIRKBY, M. J. (Ed.). Hillslope Hydrology. New York: J. Wiley, 1978. p. 1-42.

DICKINSON, W. T.; WHITELEY, H. Watershed areas contributing to runoff. IAHS Publ., v. 96, p. 12-26, 1970.

DUNNE, T. Runoff production in humid areas. U. S. Department of Agriculture Publication, ARS-41-160, 1970. 108 p.

DUNNE, T.; BLACK, R. D. Partial area contributions to storm runoff in a small New England watershed. Water Resources Research, v. 6, p. 1296-1311, 1970.

DUNNE, T.; MOORE, T. R.; TAYLOR, C. H. Recognition and prediction of runoffproducing zones in humid regions. Hydrological Sciences Bulletin, v. 20, p. 305-327, 1975.

FILL, H. D.; SANTOS, I.; FERNANDES, C.; TOCZECK, A.; OLIVEIRA, M. F. Balanço hídrico da Bacia do Rio Barigüi - PR. Revista RA'E GA, Curitiba, v. 09, p. 59-67, 2005.

FERREIRA, L. Simulação hidrológica utilizando o modelo TOPMODEL em bacias rurais: estudo de caso na bacia do Ribeirão dos Marins - Seção Manjolinho - SP. 2004. 229f. Tese (Doutorado em Engenharia Agrícola) - Universidade Estadual de Campinas, Campinas, 2004.

GBUREK, W. J.; DRUNGIL, C. C.; SRINIVASAN, M. S.; NEEDELMAN, B. A.; WOODWARD, D. E. Variable-source-area controls on phosphorus transport: Bridging the gap between research and design. Journal of Soil Water Conservation, v. 57, p. 534-543, 2002.

GORSEVSKI, P. V.; BOLL, J.; GOMEZDELCAMPO, E.; BROOKS, E. S. Dynamic riparian buffer widths from potential non-point source pollution areas in forest watersheds. Forest Ecology and Management, v. 256, p. 664-673, 2008.

HEWLETT, J. D.; HIBBERT, A. R. Factors affecting the response of small watersheds to precipitation in humid areas. International Symposium of Forest Hydrology. Oxford: Pergamon Press, 1967. p. 275-290.

KIRKBY, M. J. Inflitration, throughflow and overland flow. In: CHORLEY, R. J. Water, Earth and Man. London: Methuen, 1969. p. 215-227.

LOPES, F. C. A.; SANTOS, I. Proposta metodológica para elaboração de modelos digitais de terreno hidrologicamente consistentes. In: SIMPÓSIO DE GEOGRAFIA FÍSICA APLICADA, 13., Viçosa, 2009. Anais... Viçosa, MG: UFV, 2009. 1 CD-ROM.

MINE, M. R. M.; CLARKE, R. T. O uso do TOPMODEL em condições brasileiras: resultado preliminar. Revista Brasileira de Recursos Hídricos, Porto Alegre. v. 1, n. 2, p. 89105, 1996.

NASH, J. E.; SUTCLIFFE, J. V. River flow forecasting throught conceptual models. Journal of Hydrology, Amsterdam, v. 10, p. 282-290, 1970. 
SANTOS, I. Modelagem geobiohidrológica como ferramenta no Planejamento Ambiental: estudo da bacia hidrográfica do Rio Pequeno, São José dos Pinhais - PR. 2001. 108f. Dissertação (Mestrado em Ciências do Solo) - Universidade Federal do Paraná, Curitiba, 2001.

SANTOS, I. Monitoramento e modelagem de processos hidrogeomorlógicos: mecanismos de geração de escoamento e conectividade hidrológica. 2009. 146f. Tese (Doutorado em Geografia) - Universidade Federal de Santa Catarina, Florianópolis, 2009.

SANTOS, I.; KOBIYAMA, M. Aplicação do TOPMODEL para determinação de áreas saturadas da bacia do rio Pequeno, São José dos Pinhais, PR, Brasil. Revista Ambiente \& Água, Taubaté, v. 3, n. 2, p. 77-89, 2008.

SCHULER, A. E.; MORAES, J. M.; MILDE, L. C.; GROPPO, J. D.; MARTINELLI, L. A.; VICTORIA, R. L. et al. Análise da representatividade física dos parâmetros do TOPMODEL em uma bacia de meso escala localizada nas cabeceiras do Rio Corumbataí, São Paulo. Revista Brasileira de Recursos Hídricos, Porto Alegre. v. 5, n. 2, p. 5-25, 2000.

SILVA, R. V. da; KOBIYAMA, M. Topmodel: teoria integrada e revisão. Revista RA'E GA, Curitiba, n. 14, p. 97-110, 2007.

SUPERINTENDÊNCIA DE DESENVOLVIMENTO DE RECURSOS HÍDRICOS E SANEAMENTO AMBIENTAL DO PARANÁ - SUDERHSA-PR. Altimetria da bacia do Alto Iguaçu. Curitiba, 2000. Escala 1:10.000. Disponível em: $<$ http://www.suderhsa.pr.gov.br/modules/conteudo/conteudo.php?conteudo=99>. Acesso em: setembro de 2009.

TSUKAMOTO, Y. Storm discharge from an experimental watershed. Journal of the Japanese Forestry Society, v. 45, p. 186-190, 1963.

VARELLA, R. F.; CAMPANA, N. A. Simulação matemática do processo de transformação de chuva em vazão: estudo do modelo TOPMODEL. Revista Brasileira de Recursos Hídricos, Porto Alegre. v. 5, n. 4, p. 121-139, 2000.

WALTER, M. T.; BROOKS, E. S.; WALTER, M. S.; STEENHUIS, T. S.; SCOTT, C. A.; BOLL, J. Evaluation of soluble phosphorus transport from manure-applied fields under various spreading strategies. Journal of Soil Water Conservation, v. 56, p. 329-336, 2001.

WALTER, M. T.; WALTER M. F.; BROOKS E. S.; STEENHUIS T. S.; BOLL, J.; WEILER, K. R. Hydrologically sensitive areas: variable source area hydrology implications for water quality risk assessment. Journal of Soil Water Conservation. v. 55, p. 277-284, 2000. 